\title{
Training general practitioners contributes to the identification of palliative patients and to multidimensional care provision: secondary outcomes of an RCT
}

\author{
Bregje Thoonsen, ${ }^{1}$ Stefanie H M Gerritzen, ${ }^{1}$ Kris C P Vissers, ${ }^{1}$ \\ Stans Verhagen, ${ }^{1}$ Chris van Weel, ${ }^{2,3}$ Marieke Groot, ${ }^{1}$ Yvonne Engels ${ }^{1}$
}

\begin{abstract}
- Additional material is published online only. To view please visit the journal online (http://dx.doi.org/10.1136/ bmjspcare-2015-001031).

${ }^{1}$ Department of Anaesthesiology, Pain and Palliative Medicine, Radboudumc, Nijmegen, The Netherlands

${ }^{2}$ Department of Primary and Community Care, Radboudumc, Nijmegen, The Netherlands ${ }^{3}$ Australian Primary Health Care Research Institute, Australian National University, Canberra, Australia
\end{abstract}

Correspondence to Dr Yvonne Engels, Radboud University Medical Center, Department of Anesthesiology, Pain and Palliative Medicine, Post Box 9101, Internal Post Code 549, Nijmegen 6500 HB, The Netherlands;

yvonne.engels@radboudumc.nl

Received 29 September 2015 Revised 24 January 2016 Accepted 30 March 2016 Published Online First 18 April 2016

\section{Check for updates}

To cite: Thoonsen $B$, Gerritzen SHM, Vissers KCP, et al. BMJ Supportive \& Palliative Care 2019;9:e18.

\section{ABSTRACT}

Introduction To support general practitioners (GPs) in providing early palliative care to patients with cancer, chronic obstructive pulmonary disease or heart failure, the RADboud university medical centre indicators for PAlliative Care needs tool (RADPAC) and a training programme were developed to identify such patients and to facilitate anticipatory palliative care planning. We studied whether GPs, after 1 year of training, identified more palliative patients, and provided multidimensional and multidisciplinary care more often than untrained GPs.

Methods We performed a survey 1 year after GPs in the intervention group of an RCT were trained.

With the help of a questionnaire, all 134 GPs were asked how many palliative patients they had identified, and whether anticipatory care was provided. We studied number of identified palliative patients, expected lifetime, contact frequency, whether multidimensional care was provided and which other disciplines were involved. Results Trained GPs identified more palliative patients than did untrained GPs (median 3 vs 2; $p$ 0.046 ) and more often provided multidimensional palliative care ( $p$ 0.024). In both groups, most identified patients had cancer.

Conclusions RADPAC sensitises GPs in the identification of palliative patients. Trained GPs more often provided multidimensional palliative care. Further adaptation and evaluation of the tools and training are necessary to improve early palliative care for patients with organ failure. Trial registration number NTR2815; post results.

\section{INTRODUCTION}

Many patients at an advanced stage of cancer or other life-limiting disease suffer from problems such as pain, nausea or depressed mood. ${ }^{1-3}$ In several studies from the USA and Canada, mainly regarding patients with cancer, early palliative care increased quality of life and decreased emotional distress and useless aggressive interventions. ${ }^{4-6}$ Despite these positive findings, palliative care is still often restricted to reactive terminal care and to patients with cancer. ${ }^{7}$ However, not only do existing problems and requirements need to be addressed, future scenarios should also be considered. Early initiation of palliative care enables anticipation of future problems, needs and wishes of patients.

Also, in the Netherlands, early palliative care is not a part of daily practice. The GP is the preferred professional to coordinate palliative care $^{8}$ as most patients live at home in the final phase of their lives. Every person has his or her own gate keeping GP; also, out of hours care is arranged via the GP out-of-hours cooperatives, which, in addition, have a gate keeping function. GPs consider palliative care as an attractive and important aspect of their profession, even though it is demanding and challenging. ${ }^{9} 10$ However, they consider timely identification of patients who can benefit from anticipatory palliative care challenging, particularly in patients with chronic obstructive pulmonary disease (COPD) or chronic heart failure (CHF), as the course of these disease trajectories is difficult to predict. $^{2}{ }^{11-14}$

For these reasons we developed the RADboud university medical centre 
indicators for PAlliative Care needs (RADPAC), to support GPs in the early identification of those patients with cancer, COPD or CHF, who might profit from palliative care. ${ }^{15}{ }^{16}$ RADPAC contains indicators for GPs, used as triggers to identify a need for palliative care in these patient groups. Besides, we developed a specific training programme for GPs on using RADPAC and on how to provide structured anticipatory palliative care. The effect of this training was subsequently evaluated in a randomised controlled trial (RCT) in which over 130 GPs participated. ${ }^{17}$ We did not find a difference between the patients with cancer, COPD or CHF who died in the year after the start of the RCT in any of the groups of trained and untrained GPs in contact with the out-of-hours GP cooperative in the patients' final months of life, contacts with their own GP, hospitalisations or place of death. However, of those patients of trained GPs, who died in the year after the training, only one-third had been identified by their GPs as being palliative patients. Those patients actually identified had had significantly more contact with their GP (13 vs 7.48/month), had undergone less hospitalisations ( $14 \%$ vs $32 \%)$, and more often died at home (67 vs $45 \%$ ) and less often in hospital (14 vs 32\%) than all the other deceased patients. ${ }^{17}$ These retrospectively collected outcome measures of deceased patients were limited to short-term effects, did not provide insights into the palliative care provided, and did not provide insights into the effects of the several components of the training.

For those reasons, 1 year after start of the RCT, we studied whether trained GPs at that time cared for a larger number of palliative patients than the untrained GPs, and whether the care provided was more often multidimensional (exploration of somatic, social and financial, caregiving and activities of daily living, and existential and psychological problems and needs) and multiprofessional (different types of healthcare professionals involved). Finally, in the untrained GP group, we studied the short-term effect of providing the RADPAC.

\section{METHODS}

\section{Design}

We performed a survey 1 year after the start of an RCT, begun in 2010, to compare trained (intervention) and untrained (control) GPs as additional, secondary outcomes.

\section{Ethical procedures}

The study was conducted after approval of the research ethics committee of the Radboud University Nijmegen Medical Centre in accordance with the Medical Research Involving Human Subjects Acts (WMO) (registration number 2007/205), and registered in the Netherlands Trial Register (NTR2815).

\section{Participants}

In total, 134 GPs who had participated in the RCT 1 year prior received a questionnaire by mail in Spring 2011. Of them, 57 GPs (intervention group) had been trained 1 year prior in the early identification of their palliative patients, using the RADPAC, and in providing structured proactive palliative care in their daily practice, while the 77 untrained GPs had provided care as usual (control group).

\section{Intervention that the trained GPs had received $\mathbf{1}$ year prior}

One year before this survey, those GPs allocated to the intervention group had undergone a $5 \mathrm{~h}$ group training session in early identification and proactive palliative care planning. During this training, they received and practised with the following tools, developed within our research group: (1) the RADPAC, a tool with specific indicators to identify patients with, respectively, COPD, CHF and cancer, who might profit from palliative care, ${ }^{16}$ and (2) a proactive palliative care planning card, the 'problems and needs square': a tool to make a structured proactive care plan in which actual as well as possible future problems (somatic, social and financial, caregiving and activities of daily living, and existential and psychological), dying scenarios and patient's' wishes and needs are considered. The GPs of the trained group were invited to apply this knowledge and these tools in their daily practice, and thus to identify palliative patients and to make a proactive palliative care plan for each of them (see online supplementary appendices 1 and 2).

Regarding each identified palliative patient, the trained GPs were offered an individual coaching session by phone, with a consultant specialised in palliative care. During this session, they discussed the anticipatory care plan the GP had prepared, and adapted it if needed. Finally, all trained GPs were offered two additional face-to-face peer group sessions in which experiences could be exchanged and they could practise communicating end of life aspects on simulated patients. ${ }^{16}$ The GPs in the control group had received neither training nor intervention.

\section{Data collection}

Baseline characteristics, demographics and practice characteristics of the GPs had been collected 1 year prior, at the start of the RCT. These were age, gender, fulltime equivalent work experience, years of experience as a GP ( $\leq 1$ year, 1-5 years, 5-9 years, $\geq 10$ years), their interest in palliative care (numeric rating scale (NRS) from 0 , no interest at all, to 10, very interested) and their self-efficacy in providing palliative care (NRS from 0, no confidence at all, to 10 , very confident). Also collected was information on the kind of practice (solo, duo or group) and the degree of urbanisation of their general practice as well 
as their mean patient list and the estimated number of palliative patients per year.

\section{Effects on the provision of palliative care, 1 year after the training took place}

With the help of a questionnaire, all GPs (trained or untrained) were asked how many palliative patients they currently cared for. They did not receive a definition of palliative care, but they did know that the research project concerned identification of and proactive care planning for palliative patients with the GP as coordinator. Of each of these patients, they were asked to provide gender, age, disease (cancer, COPD, $\mathrm{CHF}$, other), expected remaining lifetime (in weeks), their current type and number of contacts with the patient (per month; respectively, by phone, consultation or home visits); whether a key information summary had been handed over to the GP out-of-hours cooperative; which type of problems they had explored (somatic, social and financial, care giving and activities of daily living, spiritual and psychological aspects; yes/ no questions) and the type of other involved disciplines in the care for the patient (physiotherapist, spiritual caregiver, social worker, medical specialist, palliative care consultant, psychologist, nursing aid at home, volunteers; yes/no questions).

\section{Short-term effects of applying RADPAC}

Three weeks after having received the questionnaire, GPs in the control group who had responded to the first questionnaire were sent the RADPAC, which they had never seen before. ${ }^{16}$ They were asked to complete another, similar questionnaire again, in which they could add those palliative patients they recognised as being palliative, after being triggered by the RADPAC indicators.

\section{Statistical analysis}

To study differences between trained and untrained GPs, we analysed, using the data of the first questionnaire, the number of palliative patients the GPs were currently aware of and their characteristics, as well as the estimated remaining lifetime (mean and \% $>4$ weeks)-whether or not the GP out-of-hours cooperative was informed-the number and type of palliative care domains that were considered per patient as well as the percentage of patients who had had a multidimensional problem and needs exploration (operationalised as three or more domains being explored) and number and type of involved disciplines. Additional palliative patient data collected from the untrained GPs after having received the RADPAC were not included in these analyses but described separately.

Statistical analyses were performed with the use of SPSS software, V.20.0. Descriptive statistics were used to calculate frequencies, means and SDs of the study variables. Differences between trained and untrained
GPs, and between patients of trained and of untrained GPs, were assessed with the use of Fisher Exact tests for categorical variables, as some cells counted less than 5 and, because of the relatively small number of patients, with the non-parametric Mann Whitney tests for continuous variables. As these data concerned secondary outcomes of an RCT, no power calculation was made.

\section{RESULTS}

In total, $12 / 57$ trained GPs (21\%) from the intervention group and 28/77 (36\%) of the control group filled in the questionnaire. Responding trained and untrained GPs had about the same mean age (45 and 48 years, respectively; table 1). Of the trained GPs, $75 \%$ were males, and of the untrained GPs, $68 \%$ were males. Besides, $42 \%$ of the trained GPs and $63 \%$ of the untrained GPs worked fulltime. A lower percentage of the trained GPs had 10 or more years' experience (67 vs $78 \%$ ). Their interest in palliative care was the same (both NRS 8) as well as their self-efficacy in providing it (both NRS 7). Most trained GPs worked in a dual practice (58\%), and the untrained GPs more often in a solo $(32 \%)$ or group practice or healthcare centre (39\%). Of the trained GPs, $67 \%$ worked in an urban region, while this figure was $50 \%$ for the untrained GPs. The patient list of the practice where the trained GPs worked was higher than the list of the practices of the untrained GPs (respectively, 3491 and 2787 patients). Almost all GPs in both groups were estimated to have between 0 and 5 palliative patients per year ( $75 \%$ and $86 \%$, respectively).

Of the non-responders, the percentage of women was higher, they worked in larger practices with a longer patient list and with more palliative patients (table 1).

In total, all GPs together had identified 119 (46 plus 73) palliative patients (figure 1). The trained GPs had identified a median of 3 palliative patients, which was significantly higher than the median of 2 patients

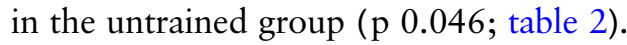

Of the palliative patients, the median age was about the same in both groups (74.5 vs 70 years; $p 0.064)$ as well as their gender ( $44 \%$ vs $55 \%$ males; p 0.544). In both groups, the primary diagnosis was most often cancer $(76 \%$ and $80 \%$; $p$ 0.630). The category 'other' diseases (respectively, 20\% and 19\%) mostly concerned a combination of either cancer and CHF, CHF and COPD, or COPD and cancer. A few times, other diagnoses such as dementia, amyotrophic lateral sclerosis, a cerebrovascular accident or chronic kidney failure were mentioned.

The median estimated remaining lifetime of the palliative patients was about the same in trained and untrained GPs (16 vs 20 weeks; p 0.728). The percentage of expected remaining lifetime being $\geq 4$ weeks was non-significantly higher in patients of the trained GPs (94\% vs 64\% of the patients; $\mathrm{p} 0.062$ ). 
Table 1 General practitioners and practice characteristics responders and non responders

\begin{tabular}{|c|c|c|c|c|}
\hline GPs participating in the RCT 1 year prior & $\begin{array}{l}\text { Intervention }(n=12) \\
\text { Responders }\end{array}$ & Control $(n=28)$ & $\begin{array}{l}\text { Intervention }(n=45) \\
\text { Non-responders }\end{array}$ & Control $(n=49)$ \\
\hline Age in years mean (SD) & $45(8)$ & $48(8)$ & $49(8)$ & $48(8)$ \\
\hline Male gender $n(\%)$ & $9(75.0)$ & $19(67.9)$ & $27(60.0)$ & $26(53.1)$ \\
\hline \multicolumn{5}{|l|}{ Working hours $\mathrm{n}(\%)$} \\
\hline Fulltime & $5(41.7)$ & $17(63.0)$ & $21(46.7)$ & $25(51.0)$ \\
\hline Part-time & $7(58.3)$ & $10(37.0)$ & $24(53.3)$ & $24(49.0)$ \\
\hline \multicolumn{5}{|l|}{ Years of experience $n(\%)$} \\
\hline$\leq 1$ & $0(0)$ & $0(0)$ & $1(2.3)$ & $1(2.0)$ \\
\hline $1-5$ & $2(16.7)$ & $2(7.4)$ & $3(6.8)$ & $7(14.3)$ \\
\hline $6-10$ & $2(17.7)$ & $4(14.8)$ & $9(20.5)$ & $6(12.2)$ \\
\hline$\geq 10$ & $8(66.7)$ & $21(77.8)$ & $31(70.5)$ & $35(71.4)$ \\
\hline $\begin{array}{l}\text { Interest in pall care* } \\
\text { NRS } 0-10 \text { (IQR) }\end{array}$ & $8.0(0.0)$ & $8.0(1.0)$ & $8.0(1.0)$ & $8.0(2.0)$ \\
\hline $\begin{array}{l}\text { Estimation of self-efficacy in palliative care provisiont } \\
\text { NRS 0-10 (IQR) }\end{array}$ & $7.0(2.0)$ & $7.0(0.5)$ & $7.0(0.8)$ & $7.0(1.0)$ \\
\hline \multicolumn{5}{|l|}{ Practice type $n(\%)$} \\
\hline Single-handed & $2(16.7)$ & $9(32.1)$ & $7(15.6)$ & $10(20.4)$ \\
\hline Dual & $7(58.3)$ & $8(28.6)$ & $21(46.7)$ & $18(36.7)$ \\
\hline Group or health centre & $3(25.0)$ & $11(39.3)$ & $17(37.8)$ & $21(42.9)$ \\
\hline \multicolumn{5}{|l|}{ Urbanisation degree $\mathrm{n}(\%)$} \\
\hline Urban & $8(66.7)$ & $14(50)$ & $22(48.9)$ & $32(65.3)$ \\
\hline Rural & $4(33.3)$ & $14(50)$ & $23(51.1)$ & $17(34.7)$ \\
\hline Patient list mean (SD) & $3491(1579)$ & $2787(1389)$ & $3602(2101)$ & $3024(1535)$ \\
\hline \multicolumn{5}{|l|}{ Estimated number of palliative patients/year $n(\%)$} \\
\hline$\leq 2$ & $4(33.3)$ & $13(46.4)$ & $3(3.8)$ & $6(12.2)$ \\
\hline $3-5$ & $5(41.7)$ & $11(39.3)$ & $22(50.0)$ & $24(49.0)$ \\
\hline $6-9$ & $3(25)$ & $3(10.7)$ & $16(36.4)$ & $16(32.7)$ \\
\hline$\geq 10$ & $0(0)$ & $1(3.6)$ & $3(6.8)$ & $3(6.1)$ \\
\hline
\end{tabular}

*Interest in palliative care: numeric rating scale (NRS) from 0 (no interest at all) to 10 (extremely interested). tEstimation of self-efficacy: NRS from 0 (not capable at all) to 10 (extremely capable).

GPS, general practitioners; RCT, randomised controlled trial.

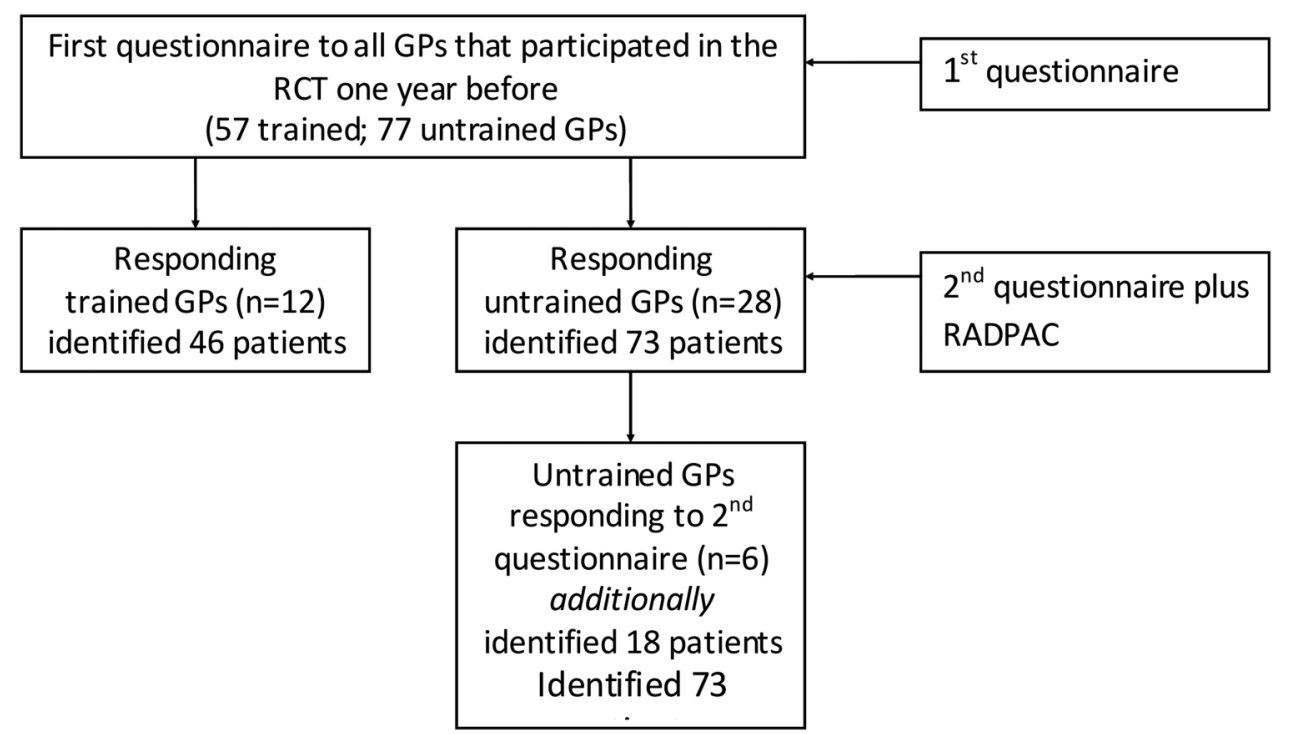

Figure 1 Prisma flow chart of involved GPs and identified palliative patients. GP, general practitioner; RCT, randomised controlled trial; RADPAC, RADboud university medical centre indicators for PAlliative Care needs. 
Table 2 Characteristics of identified palliative patients and palliative care they received

\begin{tabular}{|c|c|c|c|}
\hline & Patients of trained GPs $(n=46)$ & Patients of untrained GPs $(n=73)$ & P Value \\
\hline Number of identified patients median (min-max) & $3(2-8)$ & $2(1-7)$ & 0.046 \\
\hline Age in years median (min-max) & $74.5(39-96)$ & $70(10-98)$ & 0.064 \\
\hline Male gender* n (\%) & $20(43.5)$ & $35(54.7)$ & 0.544 \\
\hline Missing & 5 & 9 & \\
\hline Primary diagnosis* n (\%) & & & 0.630 \\
\hline Cancer & $34(75.6)$ & $58(79.5)$ & \\
\hline COPD & $2(4.4)$ & $1(1.4)$ & \\
\hline CHF & $0(0)$ & $0(0)$ & \\
\hline Othert & $9(20)$ & $14(19.2)$ & \\
\hline Expected lifetime weeks median (min-max) & $16(2-100)$ & $20(1-82)$ & 0.728 \\
\hline Expected remaining lifetime $\geq 4$ weeks* $n(\%)$ & $43(93.5)$ & $47(64.4)$ & 0.062 \\
\hline Missing<s> expected life time $n(\%)$ & $3(7)$ & $14(19)$ & \\
\hline Current monthly contact frequency median (min-max) & $4(0-24)$ & $2(1-36)$ & 0.690 \\
\hline \multicolumn{4}{|l|}{$\begin{array}{l}\text { Contact(s) per patient with own } \\
\text { GP last month } \geq 1 \text { monthly } n(\%)\end{array}$} \\
\hline By phone office hours & $25(52.1)$ & $19(27.5)$ & 0.025 \\
\hline By consultation office hours & $14(27.5)$ & $12(17.6)$ & 0.243 \\
\hline Home visits office hours & $41(80.4)$ & $60(85.7)$ & 0.739 \\
\hline GP informed out-of-hour service about patient* $\mathrm{n}(\%)$ & $26(56.5)$ & $45(61.6)$ & 0.324 \\
\hline \multicolumn{4}{|l|}{ Type of dimensions* $n(\%)$} \\
\hline Somatic & $25(54.3)$ & $39(53.4)$ & 0.852 \\
\hline Social and financial & $7(15.2)$ & $7(9.6)$ & 0.564 \\
\hline Activities of daily living (ADL) & $24(52.2)$ & $30(41.1)$ & 0.455 \\
\hline Spiritual and psychological & $19(41.3)$ & $17(23.3)$ & 0.103 \\
\hline Number of dimensions median (minimum-maximum) & $2(0-4)$ & $1(0-4)$ & 0.266 \\
\hline \multicolumn{4}{|l|}{ Number of dimensions* $\mathrm{n}(\%)$} \\
\hline 0 dimensions & $20(43.5)$ & $31(42.5)$ & \\
\hline 1 dimension & $1(0.02)$ & $7(0.1)$ & \\
\hline 2 dimensions & $5(0.3)$ & $20(43.5)$ & \\
\hline$\geq 3$ dimensions & $20(43.5)$ & $15(20.5)$ & 0.024 \\
\hline \multicolumn{4}{|l|}{ Type of disciplines* yes (\%) } \\
\hline Physiotherapist & $1(2)$ & $7(10)$ & 0.141 \\
\hline Spiritual caregiver & $2(4)$ & $5(7)$ & 0.700 \\
\hline Social work & $0(0)$ & $2(3)$ & 0.515 \\
\hline Medical specialist & $34(74)$ & $49(67)$ & 0.416 \\
\hline Palliative care consultant & $3(7)$ & $7(10)$ & 0.738 \\
\hline Psychologist & $0(0)$ & $2(3)$ & 0.515 \\
\hline Home care (nurse) & $19(41)$ & $21(29)$ & 0.323 \\
\hline Volunteers & $2(4)$ & $12(16)$ & 0.043 \\
\hline Number of disciplines median (min-max) & $2(0-4)$ & $2(0-5)$ & 0.849 \\
\hline
\end{tabular}

${ }^{*}=$ Fisher exact for categorical variables. Other $p$ values are Mann Whitney tests.

tCombination of CHF, COPD and cancer or another disease such as dementia, neurological causes or kidney failure. Data are mean (SD) or $\mathrm{n}(\%)$. Some percentages do not sum to $100 \%$ because of rounding.

$\mathrm{CHF}$, chronic heart failure; COPD, chronic obstructive pulmonary disease; GP, general practitioner.

Bold indicates statistically significant $p$-values.

We found no significant difference between trained and untrained GPs in the percentage of patients of whom the GP out-of-hours cooperative was informed (57\% vs 62\%; p 0.324).

The median number of total contacts with their own GP was about the same in both groups (4 vs 2; $\mathrm{p}$ 0.690). Almost all patients had had at least one monthly contact with their GP. The percentage of patients who had had at least one contact by phone was higher in the trained GP group (52\% vs $28 \%$; p 0.025). The percentage of patients who had had at least one monthly consultation did not differ significantly between either group ( $28 \%$ vs $18 \%$; p 0.243 ), nor did the percentage of patients with at least one home visit per month (80\% and $86 \%$; p 0.739).

The median number of explored dimensions (somatic, social and financial, activities of daily living (ADL), and spiritual and psychological) did not differ 
significantly ( 2 and 1 , respectively; $p$ 0.266), but trained GPs more often explored $\geq 3$ dimensions ( $44 \%$ vs $21 \%$; p 0.024). In the patients of the trained GPs, the spiritual and psychological dimension was twice as often explored, but this difference was not significant ( $41 \%$ vs $23 \%$; $p$ 0.103).

The median number of other involved disciplines was the same in both groups $(2 ; \mathrm{p}$ 0.849). In the care of patients of the untrained GPs, volunteers were significantly more often involved (4\% and 16\%; $\mathrm{p} 0.043$ ).

Three weeks after the first questionnaire, the untrained GPs who had responded to the first questionnaire received the RADPAC, and were asked to use it and to then complete a similar questionnaire again, adding patients additionally identified as being palliative. Six untrained GPs $(6 / 28=21 \%$ of the GPs who responded to the first questionnaire) also completed this questionnaire and became aware of a mean of 3 additional palliative patients (table 3). These six GPs had already identified a mean of 1.8 patients after the first questionnaire. Of these 18 patients, 5 patients $(28 \%)$ had chronic organ failure.

\section{DISCUSSION}

One year after start of an RCT in which GPs in the intervention group were trained in identifying their palliative patients and in structured, proactive palliative care, trained GPs were aware of significantly more palliative patients in their practice and more often provided multidimensional care. With the help of an additional questionnaire a few weeks later, we also found that untrained GPs who received the RADPAC identified additional palliative patients. ${ }^{16}$

The percentage of patients with an expected remaining lifetime of $\geq 4$ weeks was non-significantly higher in the trained GP group. However, as we performed a cross-sectional study and did not follow-up when these patients actually died, we do not know how long the actual survival was for and whether the correctness of the estimation differed between trained and untrained GPs. Twenty per cent of the untrained GPs did not complete the question about the remaining lifetime expectancy, while this figure was only $7 \%$ in the trained GPs. In the untrained GPs, a prognostic paralysis might have contributed to this large number of missings. ${ }^{18}$ Two other studies also showed that it is hard for GPs to provide such figures. ${ }^{9}$ 19-23

Of the trained GPs, twice as many palliative patients had had multidimensional problems and needs assessment, meaning that at least three dimensions (somatic, social and financial, ADL, and spiritual and psychological) were explored. Particularly, exploration of the spiritual and psychological dimension contributed to this difference. A Dutch study of Van der Brandt showed that psychological and spiritual aspects of care need more attention in the last days of dying patients. ${ }^{24-26}$ Although person-centred, integrated care belongs to the core values of GPs and the importance of the biopsychosocial model in the chronic care context has been recognised by all relevant Dutch stakeholders, this ideal appeared to be poorly embed$\operatorname{ded}^{8}$; our results suggest that this is also true for palliative care. This is not a surprise, since guidelines and standards as well as reimbursement of care expenditures are mainly based on biomedical outcome indicators, ignoring the psychological or spiritual aspects.

Most often, the somatic domain was explored by both the trained as well as the untrained GPs. This finding is in line with their medical background. However, in contrast to a study by Meijler et al, ${ }^{10}$ which showed that a workshop in palliative care mainly improved attention and knowledge of the somatic dimension, the trained GPs in our study did not differ from the untrained GPs in this aspect. ${ }^{27}$ As the somatic domain was only explored in half of the identified patients, this also implies that for half of the patients this was not carried out, although it is known that many patients at an advanced stage of cancer, ${ }^{1} \mathrm{COPD}^{2}$ or $\mathrm{CHF}$, suffer multiple problems and many patients do not report these problems spontaneously. ${ }^{28} 29$

No significant difference between trained and untrained GPs was found in the number of disciplines involved in the palliative care of their patients. In about two-third of the patients of the trained as well

Table 3 Untrained GPs who completed 1st and 2nd* questionnaires

\begin{tabular}{|c|c|c|c|c|c|c|c|}
\hline Untrained GP $(n=6)$ id & $\begin{array}{l}\text { identifiedt palliative } \\
\text { patients 1st } \\
\text { questionnaire (n) }\end{array}$ & Cancer & $\begin{array}{l}\text { Identified } ¥ \text { palliative } \\
\text { patients } 2 \text { nd* } \\
\text { questionnaire }(n)\end{array}$ & Cancer & COPD & CHF & $\begin{array}{l}\text { Total }(n) \text { of identified } \\
\text { patients }\end{array}$ \\
\hline $\bar{A}$ & 1 & 1 & 1 & 1 & & & 2 \\
\hline B & 1 & 1 & 2 & 1 & & 1 & 3 \\
\hline C & 1 & 1 & 2 & 1 & & 1 & 3 \\
\hline $\mathrm{D}$ & 4 & 4 & 6 & 5 & & 1 & 10 \\
\hline$E$ & 3 & 3 & 3 & 3 & & & 6 \\
\hline $\mathrm{F}$ & 1 & 1 & 4 & 2 & 1 & 1 & 5 \\
\hline
\end{tabular}

${ }^{*}$ In this similar questionnaire, they were asked to mention additionally identified patients, triggered by the RADPAC that they had received alongside. tThese GPs only identified those patients with cancer who were mentioned in the first questionnaire. 
as untrained GPs, a medical specialist was involved. In many cases, palliative patients continue to visit the medical specialist, even when no life prolonging treatment options are available anymore. Weeks et $a l^{30}$ found that many patients with incurable cancer receiving chemotherapy did not understand that chemotherapy is unlikely to be curative. This wrong perception compromises their ability to make informed treatment decisions. It implies that patients often receive treatments for too long. Improving interdisciplinary collaboration between the medical specialist and GP might contribute to shared decision-making regarding advantages and disadvantages of treatment options.

The six untrained GPs who responded to the second questionnaire, after having received the RADPAC, became aware of a mean of 3 additional palliative patients, which was even more than the 1.8 patients they mentioned in the first questionnaire, suggesting that RADPAC contributes to the identification of palliative patients. A quarter of these additional palliative patients had COPD or CHF, which is a higher percentage than in the first questionnaire of the trained as well as untrained GPs, and more in line with Dutch mortality data of these diseases. ${ }^{31}$

These positive effects indicate that RADPAC might be an answer to the need for tools to identify palliative patients at an earlier stage. ${ }^{16} 32-34$

\section{Strengths and weaknesses}

This is one of the first studies that, using an additional question within an RCT, evaluated long-term effects of training in which GPs in the intervention group had been trained in identifying patients in need of palliative care and in providing structured, proactive palliative care. ${ }^{15}{ }^{16}$ In this study, we also tried to make a distinction between the effects of the RADPAC and those of training in providing multidimensional palliative care.

Unfortunately, the response rate was extremely low. Regarding the trained GPs, the response rate was only $21 \%$. Of the untrained GPs, the response rate was $36 \%$, but regarding the second questionnaire in this group, this figure was also low (21\%). Probably mainly the most motivated GPs will have responded, which might have influenced the results. Another questionnaire regarding the primary and secondary outcomes of the RCT, sent to the same GPs at the same time and described elsewhere, ${ }^{17}$ resulted in a response rate of $57 \%$, which is much higher. An explanation might be that the practice assistant could complete that questionnaire, which concerned deceased patients, while the questionnaire about current palliative patients had to be completed necessarily by the GP. The trained GPs had completed a considerable number of forms during the intervention period, the year before. Voting with their feet as a result of fatigue regarding being asked again to complete a questionnaire, and time investment of all GPs, might have been barriers to completing it. ${ }^{35}$ The GPs received this questionnaire in the run-up to a national protest action, in which most Dutch GPs signed a manifest, 'Het roer moet om' (A change of direction) against the growing workload of administration, forms and obligations. ${ }^{36}$ This probably will have contributed to the low response rate. Another limitation is that we had not asked how many palliative patients the GPs cared for at baseline. Owing to this, the low response rate and the fact that these data were secondary outcomes that were not used in a power calculation, all findings should be interpreted with caution.

\section{CONCLUSIONS}

This study shows that a training programme in proactive palliative care provision and in using RADPAC to identify palliative patients even 1 year later improves the number of identified palliative patients. Additionally, multidimensional care, which could, particularly, be contributed to more attention given to the spiritual and psychological dimension, was more often offered by trained GPs.

Untrained GPs who 3 weeks after the first questionnaire received the RADPAC and were asked to consider their patient list again to identify additional palliative patients, in total identified more patients than the trained GPs; and identified a larger percentage of patients with COPD or CHF. This indicates that, although a training programme in using the RADPAC still positively influences awareness of palliative patients 1 year later, RADPAC seems to have the largest effect immediately after applying it, and particularly in detecting patients with COPD or CHF in need of palliative care. As RADPAC is restricted to cancer, COPD and CHF, we recommend extension with indicators for other life-limiting diseases, as has been carried out with other tools. ${ }^{20}$

Finally, we recommend an implementation trajectory in which GPs as well as medical specialists are involved, in which timely identification of palliative patients is an ongoing process.

Acknowledgements The authors thank all the participating GPs.

Contributors BT designed the intervention, included the participants, wrote the protocol, developed the tools, wrote the statistical analysis plan and drafted and revised the paper. SG cleaned and analysed the data and wrote the first version of the paper. KV co-designed the intervention, and drafted and revised the paper. SV co-designed the intervention, monitored data collection and drafted the paper. $\mathrm{CvW}$ co-designed the intervention and revised the paper. MG co-designed the intervention, developed the tools, monitored data collection and revised the paper. YE designed the intervention, was principal investigator, monitored data collection, wrote the statistical plan, supervised the analyses, and drafted and revised the paper.

Funding The study was financially supported by a grant from the Netherlands Organisation for Health Research and Development-ZonMw Foundation. Grant number 1150.0002 .

Competing interests None declared.

Ethics approval Research ethics committee of the Radboud University Nijmegen Medical Centre in accordance with the Medical Research Involving Human Subjects Acts (WMO) (registration number 2007/205). 
Provenance and peer review Not commissioned; externally peer reviewed.

\section{REFERENCES}

1 Teunissen SC, Wesker W, Kruitwagen C, et al. Symptom prevalence in patients with incurable cancer: a systematic review. J Pain Symptom Manage 2007;34:94-104.

2 Curtis JR. Palliative and end-of-life care for patients with severe COPD. Eur Respir J 2008;32:796-803.

3 Whellan DJ, Goodlin SJ, Dickinson MG, et al Quality of Care Committee, Heart Failure Society of America. End-of-life care in patients with heart failure. J Card Fail 2014;20:121-34.

4 Temel JS, Greer JA, Muzikansky A, et al. Early palliative care for patients with metastatic non-small-cell lung cancer. $N$ Engl $J$ Med 2010;363:733-42.

5 Bakitas M, Lyons KD, Hegel MT, et al. Effects of a palliative care intervention on clinical outcomes in patients with advanced cancer: the Project ENABLE II randomized controlled trial. JAMA 2009;302:741-9.

6 Zimmermann C, Swami N, Krzyzanowska M, et al. Early palliative care for patients with advanced cancer: a cluster-randomised controlled trial. Lancet 2014;383:1721-30.

7 Beernaert K, Cohen J, Deliens L, et al. Referral to palliative care in COPD and other chronic diseases: a population-based study. Respir Med 2013;107:1731-9.

8 van Dijk-de Vries A, Moser A, Mertens VC, et al. The ideal of biopsychosocial chronic care: how to make it real? A qualitative study among Dutch stakeholders. BMC Fam Pract 2012;13:14.

9 Groot MM, Vernooij-Dassen MJ, Crul BJ, et al. General practitioners (GPs) and palliative care: perceived tasks and barriers in daily practice. Palliat Med 2005;19:111-18.

10 Meijler WJ, Van Heest F, Otter R, et al. Educational needs of general practitioners in palliative care: outcome of a focus group study. J Cancer Educ 2005;20:28-33.

11 Hanratty B, Hibbert D, Mair F, et al. Doctors' perceptions of palliative care for heart failure: focus group study. BMJ 2002;325:581-5.

12 Goodlin SJ, Hauptman PJ, Arnold R, et al. Consensus statement: palliative and supportive care in advanced heart failure. J Card Fail 2004;10:200-9.

13 O'Leary N, Murphy NF, O'Loughlin C, et al. A comparative study of the palliative care needs of heart failure and cancer patients. Eur J Heart Fail 2009;11:406-12.

14 Jaarsma T, Beattie JM, Ryder M, et al. Advanced Heart Failure Study Group of the HFA of the ESC. Palliative care in heart failure: a position statement from the palliative care workshop of the Heart Failure Association of the European Society of Cardiology. Eur J Heart Fail 2009;11:433-43.

15 Thoonsen B, Groot M, Engels Y, et al. Early identification of and proactive palliative care for patients in general practice, incentive and methods of a randomized controlled trial. BMC Fam Pract 2011;12:123.

16 Thoonsen B, Engels Y, van Rijswijk E, et al. Early identification of palliative care patients in general practice: development of RADboud indicators for PAlliative Care Needs (RADPAC). $\mathrm{Br} J$ Gen Pract 2012;62:e625-31.

17 Thoonsen B, Vissers K, Verhagen S, et al. Training general practitioners in early identification and anticipatory palliative care planning: a randomized controlled trial. BMC Fam Pract 2015;16:126.
18 Stewart S, McMurray J. Palliative care for heart failure. BMJ 2002;325:915-16.

19 Mitchell G. How well do general practitioners deliver palliative care? A systematic review. Palliat Med 2002;16:457-64.

20 Maas EA, Murray SA, Engels Y, et al. What tools are available to identify patients with palliative care needs in primary care: a systematic literature review and survey of European practice. BMJ Support Palliat Care 2013;3:444-51.

21 Slort W, Schweitzer B, Blankenstein A, et al. Perceived barriers and facilitators for general practitioner-patient communication in palliative care: a systematic review. Pall Med 2011;25:613-29.

22 Parry R, Land V, Seymour J. How to communicate with patients about future illness progression and end of life: a systematic review. BMJ Support Palliat Care 2014;4:331-41.

23 Brandt HE, Ooms ME, Ribbe MW, et al. Predicted survival vs. actual survival in terminally ill noncancer patients in Dutch nursing homes. J Pain Symptom Manage 2006;32:560-6.

24 Brandt H, Deliens L, van der Steen JT, et al. The last days of life of nursing home patients with and without dementia assessed with the palliative care outcome scale. Pall Med 2005;19:334-42.

25 Vermandere M, De Lepeleire J, Van Mechelen W, et al. Outcome measures of spiritual care in palliative home care: a qualitative study. Am J Hosp Palliat Care 2013;30:437-44.

26 Kelly B, Varghese FT, Burnett P, et al. General practitioners' experiences of the psychological aspects in the care of a dying patient. Palliat Support Care 2008;6:125-31.

27 Schuit KW, Bender W, Meijler WJ, et al. Learning effects of a workshop in palliative cancer care for general practitioners. J Cancer Educ 1999;14:18-22.

28 Goodlin SJ. Palliative care in congestive heart failure. J Am Coll Cardiol 2009;54:386-96.

29 Chen-Scarabelli C, Saravolatz L, Hirsh B, et al. Dilemmas in end-stage heart failure. J Geriatr Cardiol 2015;12: 57-65.

30 Weeks JC, Catalano PJ, Cronin A, et al. Patients' expectations about effects of chemotherapy for advanced cancer. N Engl J Med 2012;367:1616-25.

31 Statistics Netherlands. https://www.volksgezondheidenzorg.info/ onderwerp/sterfte-naar-doodsoorzaak (accessed 2 Dec 2015).

32 Gomez-Batiste X, Martinez-Munoz M, Blay C, et al. Identifying patients with chronic conditions in need of palliative care in the general population: development of the NECPAL tool and preliminary prevalence rates in Catalonia. BMJ Support Palliat Care 2013;3:300-8.

33 Haga K, Murray S, Reid J, et al. Identifying community based chronic heart failure patients in the last year of life: a comparison of the Gold Standards Framework Prognostic Indicator Guide and the Seattle Heart Failure Model. Heart 2012;98:579-83.

34 Highet G, Crawford D, Murray SA, et al. Development and evaluation of the Supportive and Palliative Care Indicators Tool (SPICT): a mixed-methods study. BMJ Support Palliat Care 2014;4:285-90.

35 Cottrell E, Roddy E, Rathod T, et al. Maximising response from GPs to questionnaire surveys: do length or incentives make a difference? BMC Med Res Methodol 2015;15:3.

36 Manifest from Dutch general practitioners: a change of direction. hetroermoetom.nu (accessed 2 Dec 2015). 